\title{
Interests and limitations of nanoindentation for bulk multiphase material identification: Application to the $\beta$ phase of Ti-5553
}

\author{
A.F. Gerday ${ }^{a}$, M. Ben Bettaieb ${ }^{a}$, L. Duchêne ${ }^{a}$, N. Clément ${ }^{b}$, H. Diarra ${ }^{a}$, A.M. Habraken ${ }^{a}$ \\ ${ }^{a}$ ARGENCO Department, University of Liège, Chemin des Chevreuils 1, 4000 Liège, Belgium \\ ${ }^{b}$ IMAP Department, Université Catholique de Louvain-la-Neuve, Place Sainte Barbe 2, 1348 Louvain-la-Neuve, Belgium
}

\begin{abstract}
This paper focuses on the numerical modeling of nanoindentation tests. The first goal of this study is to collect essential material parameters and boundary conditions from the literature and to complete the data required to accurately model nanoindentation tests. The second goal of this study consists in validating the material parameters identified from macroscopic tensile tests of the body-centered cubic $\beta$ phase of Ti-5553, a new generation of titanium alloy. This validation is performed looking at experimental and numerical nanoindentation curves obtained for different grain orientations.
\end{abstract}

Keywords: Finite element modeling (FEM); Nanoindentation; Titanium alloy; Sensitivity analysis

\section{Introduction}

Nanoindentation is a standard experimental method for the characterization of a material at the micro/nanoscale $[1,2]$. This technique is used to measure mechanical properties such as Young's modulus or the hardness of thin films $[3,4]$ and small volumes of materials [5-8].

The main goal of this paper is to determine the material parameters and the geometrical data that must be known with a high degree of accuracy in order to accurately model realistic nanoindentation tests. Moreover, this study is also applied to validate the material parameters identified from macroscopic tests of the body-centered cubic (bcc) phase of Ti-5553. This new generation of titanium alloy is suitable for aeronautical applications due to its high strength. To achieve both goals, information collected from the literature is summarized; the present article completes this information.

\subsection{Macroscopic material parameters}

Nanoindentation has long been used to determine the Young's modulus, $E$, and the hardness, $H$, of materials. Some authors [4,9,10] have presented methods based on the Doerner and Nix [11] and Oliver and Pharr [12] studies to extract $E$ and $H$ from the unloading part of the nanoindentation curve. Nowadays, with finite-element (FE) simulations, nanoindentation can also be used to identify other material parameters. Sasaki et al. [13], for example, performed inverse modeling of load vs. displacement curves to identify elastic orthotropic parameters of thin films. Yan et al. [14] also used inverse modeling of the nanoindentation curves to determine the plastic parameters of the material (the yield stress, $\sigma_{y}$, and the strain-hardening exponent). Liu et al. [15] focused on using nanoindentation and inverse modeling to obtain the stress vs. strain relations of a copper single crystal. Pelletier [5] identified equations for the loading and unloading parts of the nanoindentation load vs. displacement curves of bulk materials, and then used these to identify the elastic and plastic parameters of the materials (Young's modulus, Poisson's ratio, tangent modulus, strain-hardening exponent, etc.).

\subsection{Microscopic analysis}

Many authors have performed FE simulations of nanoindentation with 2-D axisymmetric meshes and simple elastoplastic constitutive laws $[14,16,17,2]$. Those formulations are sufficient for the identification of standard material parameters. However, to study the influence of other parameters, such as the grain orientation, this methodology is too coarse. In this 3-D FE mesh and a crystalline plasticity law are needed. Such a law was presented and used for copper single crystal in Wang et al. [6]. In that article, Wang et al. presented a study of the dependence of nanoindentation pile-up patterns and microtextures on the crystallographic orientation. Likewise, Zaafarani et al. [18] studied the microstructure and texture below a conical indenter. An approach incorporating a mesoplastic constitutive model to determine the material properties of copper single crystal was also developed by Liu et al. [15].

Nanoindentation experiments have been physically studied by several authors. Mirshams et al. [16] observed that the grain size had a high effect on $H$ measurements, and Lilleodden et al. [3] demonstrated that the results are 
different when the material is constituted of large coarse grains or finer ones. The orientation of the grain affects the hardness $H$ but also the number of pile-ups and their symmetries as presented by Wang et al. [6] for copper. Moreover, Liu et al. [19] mentioned that the symmetry characteristics and the size of the plastic zone strongly depend on the crystal orientation.

\subsection{Sensitivity of the pile-up and sink-in patterns}

Some authors have tried to predict the appearance of pile-up or sink-in patterns near the indented imprint. Some of these $[4,10,18,20,21]$ have related the appearance of pile-up and sink-in patterns to hardening properties. Liu et al. [15] reported that the amount of pile-up is greater when the radius of the spherical indenter used is larger, but, for Wang et al. [6], the prediction of pile-up or sink-in patterns was not so obvious. According the latter reference, these patterns are due to the crystallography and the orientation of the grain indented.

Friction also affects the appearance of pile-up or sink-in patterns. Indeed, as mentioned by Liu et al. [15], Bucaille et al. [22] and Bocciarelli et al. [23], when the coefficient of friction is greater, the height of the pile-up patterns decreases. Even though the friction influences the patterns near the indented imprint, this coefficient is generally considered to be "zero" by many authors. In fact, friction does not significantly affect the nanoindentation load vs. displacement curves. This result is observed by Liu et al. [15] and by Wang et al. [6] for friction coefficients ranging from 0 to 0.3 or 0.4 .

Tabor [24] explained this result in physical terms. He proposed an analytical model to describe the evolution of the normal force as a function of the included angle of the indenter tip, for a conical indenter. He concluded that the effect of friction is small for indenters with a large angle (like Berkovich and Vickers) and this effect is more significant when the angle of the indenter $(\theta)$ is less than $60^{\circ}$. This result was also reported by Bucaille et al. [22] and Kojima et al. [25].

\subsection{Effect of indenter geometry}

To compare experimental and corresponding numerical nanoindentation results, it is important to model the experimental problem accurately in numerical simulations. Indeed, the geometry of the indenter tip must be rigorously reproduced because it has a high effect on $H$ measurements, as noted by Mirshams et al. [16], and it significantly affects the load vs. displacement results. On the micro or nanoscale, the finite (albeit minuscule) tip radius of a real sharp tip cannot be neglected [26]. Yan et al. [14] showed that an increase in the included angle leads to an increase in the maximum force obtained for the same indentation depth. Note that, as mentioned above, Liu et al. [15] found a larger pile-up for a greater included angle.

Similarly, the curvature of the tip must be accurate. Pelletier et al. [17] showed that the numerical modeling of the curvature of the indenter tip has an influence on load vs. displacement curves. He found that a more realistic model of that curvature leads to results in better agreement with experimental ones.

\subsection{Effect of contact model and mesh density}

In several FE nanoindentation curves, some bumps occur. Liu et al. [15] observed that the curve oscillations are more numerous and pronounced for simulations carried out with a coarser mesh. This observation, also mentioned by Bocciarelli et al. [23], can be explained by the FE contact model. A smoother curve is obtained with a finer mesh. Consequently, the choice of the mesh is still a compromise between accuracy and computation time.

In the next section, the constitutive laws and the hardening models used in the simulations are briefly described. Some information about the experiments performed, the material and its parameters are given. The reference parameters used in the numerical simulations are those identified for the bcc $\beta$ phase of the Ti-5553 alloy from classical tensile tests. The FE meshes are also presented. Finally, the results of the sensitivity analysis are listed. Geometrical, elastic and plastic parameters are studied. The results are commented on and compared with experimental ones and with results obtained by other authors.

\section{Constitutive laws}

The numerical nanoindentation simulations were achieved using the Lagamine FE code [27] and two constitutive laws. The first law used is a macroscopic Von Mises' elastic-plastic (EP) constitutive law. The uniaxial stress $(\sigma)$ vs. strain ( $\varepsilon$ ) relation is given by: 


$$
\begin{cases}\sigma=\varepsilon E & \sigma \leqslant \sigma_{y 1}, \\ \sigma=\varepsilon E_{T} & \sigma \geqslant \sigma_{y 2},\end{cases}
$$

where $E$ is the Young's modulus, and $E_{T}$ is the plastic modulus. Between $\sigma_{y 1}$, the lower yield stress, and $\sigma_{y 2}$, the upper yield stress, the EP transition is given by stress vs. strain coordinates. The advantage of such a law is its simplicity and thus the reduced computation time.

The second law is an elastoviscoplastic (EVP) microscopic crystal plasticity model based on the constitutive law proposed by Huang [28] and modified by Kysar [29]. In this kind of constitutive law, the crystal orientation and the activated slip systems are taken into account. The velocity gradient $\mathrm{L}$ is decomposed as:

$$
\mathbf{L}=\mathbf{D}+\mathbf{\Omega},
$$

where $\mathrm{D}$ and $\Omega$ are the symmetric and the skew parts of the velocity gradient, respectively. $\mathrm{D}$ and $\Omega$ are additively decomposed into elastic (superscript $e$ ) and plastic (superscript $p$ ) parts as:

$$
\left\{\begin{array}{l}
\mathbf{D}=\mathbf{D}^{e}+\mathbf{D}^{p} \\
\boldsymbol{\Omega}=\mathbf{\Omega}^{e}+\mathbf{\Omega}^{p}
\end{array}\right.
$$

The elastic parts correspond to elastic deformations and lattice rotations. The plastic parts represent the plastic shear slips of the lattice and are related to the plastic shear strain rate on each slip system. The plastic part of the velocity gradient $\mathrm{L}^{p}$ is then written as:

$$
\mathbf{L}^{p}=\mathbf{D}^{p}+\mathbf{\Omega}^{p}=\sum \dot{\gamma}^{(\alpha)} \mathbf{s}^{*(\alpha)} \mathbf{n}^{*(\alpha)}
$$

where $\dot{\gamma}^{(\alpha)}$ is the shear strain rate in any given slip system $\alpha, \mathbf{s}^{*(\alpha)}$ is the slip direction vector of system $\alpha$ and $\mathrm{n}^{*(\alpha)}$ is the normal vector to the slip plane of system $\alpha$ (where, for consistency, superscript $*$ indicates that the vectors are taken in the deformed configuration following lattice stretching and rotation). The increment of the shear stress in system $\alpha$ is then prescribed through:

$$
\dot{\tau}^{(\alpha)}=\mathbf{n}^{*(\alpha)}\left[\mathbf{C}: \mathbf{D}^{e}-\mathbf{D}^{e} \cdot \boldsymbol{\sigma}+\boldsymbol{\sigma} \cdot \mathbf{D}^{e}\right] \mathbf{s}^{*(\alpha)},
$$

where $\mathrm{C}$ is the elastic stiffness tensor and $\sigma$ is the Cauchy stress tensor.

The shear strain rate $\dot{\gamma}^{(\alpha)}$ of the $\alpha$ th slip system in a rate-dependent crystalline solid is determined by the corresponding resolved shear stress $\tau^{(\alpha)}$ as proposed by Hutchinson [30]:

$$
\dot{\gamma}^{(\alpha)}=\dot{a}^{(\alpha)}\left|\frac{\tau^{(\alpha)}}{\tau_{c}^{(\alpha)}}\right|^{n} \operatorname{sgn}\left(\tau^{(\alpha)}\right),
$$

where $\dot{a}^{(\alpha)}$ is the reference strain rate on slip system $\alpha, \tau_{c}^{(\alpha)}$ is the current critical resolved shear stress, and $n$ is linked to the material strain rate sensitivity. The critical resolved shear stress $\tau_{c}^{(\alpha)}$ develops with the evolution of slips on active slip systems due to the accumulation of dislocations in a crystal through the relation:

$$
\dot{\tau}_{c}^{(\alpha)}=\sum_{\beta} h_{\alpha \beta} \dot{\gamma}^{(\beta)}
$$

where $h_{a \beta}$ are the slip hardening moduli, the sum of which ranges over all activated slip systems. Here, two 
hardening matrices are implemented. The first one is the hardening model of Pierce, Needleman and Asaro (hereafter the PAN model) [28]:

$$
\left\{\begin{array}{l}
h_{\alpha \alpha}=h(\gamma)=h_{0} \operatorname{sech}^{2}\left|\frac{h_{0} \gamma}{\tau_{s}-\tau_{0}}\right| \\
h_{\alpha \beta}=q h(\gamma) \quad(\alpha \neq \beta)
\end{array}\right.
$$

where $h_{0}$ is the initial hardening modulus, $\tau_{0}$ is the initial value of $\tau_{\mathrm{c}}^{\left({ }^{(\alpha)}\right.}$ and $\tau_{s}$ is the saturation value, "sech" is the hyperbolic secant function and $\mathrm{y}$ is the Taylor cumulative shear strain on all slip systems:

$$
\gamma=\sum_{\alpha} \int_{0}^{t}\left|\dot{\gamma}^{(\alpha)}\right| d t
$$

The parameter $q=1$ corresponding to isotropic hardening is chosen here.

The second hardening matrix was as defined by Bassani and $\mathrm{Wu}$ (hereafter the $\mathrm{BW}$ model) [31]. Its expression depends on the shear strains $y^{(\alpha)}$ of all slip systems [28]:

$$
\left\{\begin{array}{l}
h_{\alpha \alpha}=\left\{\left(h_{0}-h_{s}\right) \operatorname{sech}^{2}\left|\frac{\left(h_{0}-h_{s}\right) \gamma^{(\alpha)}}{\tau_{s}-\tau_{0}}\right|+h_{s}\right\} G\left(\gamma^{(\beta)} ; \quad \beta \neq \alpha\right), \\
h_{\alpha \beta}=q h_{\alpha \alpha} \quad(\alpha \neq \beta),
\end{array}\right.
$$

with

$$
G\left(\gamma^{(\beta)} ; \quad \beta \neq \alpha\right)=1+\sum_{\beta \neq \alpha} f_{\alpha \beta} \tanh \left(\frac{\gamma^{(\beta)}}{\gamma_{0}}\right)
$$

where $h_{0}, \tau_{0}$ and $\tau_{s}$ have the same meaning as in the PAN model. The parameter $h_{s}$ is the hardening modulus during easy glide within the first stage of hardening, $y^{(\alpha)}$ (or $\left.y^{(\beta)}\right)$ is the total shear strain in slip system $\alpha($ or $\beta$ ) and $y_{0}$ is the amount of slip after which the interaction between slip systems reaches the peak strength, and each component $f_{\alpha \beta}$ represents the magnitude of the strength of an interaction between slip system $\alpha$ and $\beta$. In this model, $q=0$ is chosen. It corresponds to diagonal hardening. The function $G$ deals implicitly with crosshardening that occurs between slip systems during the second stage of hardening [32].

\section{Experiments}

Nanoindentation tests were performed on a Hysitron Triboscope mounted on a Park Autoprobe CP atomic force/scanning tunnelling microscope. A three-sided Berkovich diamond indenter tip was used with a total included angle of $142.3^{\circ}$. As mentioned by several authors [14,17], indentations using a Berkovich indenter result in the same force vs. displacement curve as a conical indenter with a half apex angle $\theta=70.3^{\circ}$. This is the reason why various authors have preferred to simplify numerical modeling using a 2-D axisymmetric mesh with a conical indenter instead of a 3-D Berkovich one. However, this approximation does not permit researchers to study microscopic parameters.

In the present 3-D simulations, the indenter is modeled as a rigid tool. This is justified as the diamond indenter has a Young's modulus of $1000 \mathrm{GPa}$, an order of magnitude higher than that of the $\beta$ phase of Ti-5553, which is around $85 \mathrm{GPa}$.

Table 1: Parameters optimized for the $\beta$ phase of Ti-5553: Young's modulus E, Poisson's ratio $v$, yield strength $\sigma_{y}$ and tangent modulus $E_{T}$.

\begin{tabular}{llll}
\hline $\boldsymbol{E}(\mathbf{M P a})$ & $\boldsymbol{V}$ & $\boldsymbol{\sigma}_{\boldsymbol{y}}(\mathbf{M P a})$ & $\boldsymbol{E}_{\boldsymbol{T}}(\mathbf{M P a})$ \\
\hline 85,000 & 0.31 & 595 & 1388.9 \\
\hline
\end{tabular}




\section{Material and parameters}

Since nanoindentation simulations and tests focused on the $\beta$ phase behavior, a $\beta$-rich micro structure was chosen, obtained by a heat treatment close to the $\beta$ transus followed by a water quench. The material studied is the bcc $\beta$ phase of Ti-5553. The parameters of the EP constitutive law for this phase, given in Table 1, were optimized using inverse modeling on tensile tests performed on $100 \% \beta$ material [33,34]. This identification was carried out using the program Optim of the Lagamine FE code based on Levenberg Marquardt's algorithm [35].

The corresponding identified parameters of the microscopic crystal plasticity-based constitutive law and the two hardening models are given in Table 2.

When not mentioned in the following nanoindentation simulations, a friction coefficient of $\mu=0.2$ was chosen.

Table 2: Identified crystal plasticity parameters for the PAN and BW models for the $\beta$ phase of Ti-5553.

\begin{tabular}{lllll}
\hline & PAN model & BW model & \\
\hline Elastic constants & $E$ & $85,000 \mathrm{MPa}$ & $E$ & $85,000 \mathrm{MPa}$ \\
& $V$ & 0.35 & $\mathrm{~V}$ & 0.35 \\
Hardening & $\dot{\mathrm{a}}$ & 0.0001 & $\dot{\mathrm{a}}$ & 0.001 \\
parameters & $n$ & 19.3 & $n$ & 50 \\
& $h_{0}$ & $13,120 \mathrm{MPa}$ & $h_{0}$ & $7482 \mathrm{MPa}$ \\
& $\tau_{0}$ & $300 \mathrm{MPa}$ & $\tau_{0}$ & $300 \mathrm{MPa}$ \\
& $\tau_{s}$ & $353 \mathrm{MPa}$ & $\tau_{s}$ & $304 \mathrm{MPa}$ \\
& & & $\gamma_{0 \alpha \alpha}$ & 0.00091 \\
& & & $\gamma_{0 \alpha \beta}$ & 0.000314 \\
& & & $f_{\alpha \alpha}$ & 14.6 \\
& & & $h_{s}=A \ln (\dot{\varepsilon})+B$ & $A=-1.959$ \\
& & & $B=-14.766$ \\
\hline
\end{tabular}

\section{FE meshes}

Many authors use 2-D axisymmetric FE meshes because the computation time is considerably reduced which makes the characterization of the usual material parameters possible. However, in this work, to study other parameters such as the grain orientation, several 3-D meshes were defined to perform numerical nanoindentation simulations (Fig. 1). The three meshes are similar except in the central zone where the indentation is performed. The first mesh (a), the coarser one, contains 1044 eight-node brick elements. The second one (b), more refined, is composed of 1772 elements. The third and last mesh (c), well refined in the indented region, contains 4544 elements. In each mesh, contact elements [36] with nine integration points are placed in the central zone of the top surface of the mesh.

Fig. 1: (Left) Mesh used for nanoindentation simulations (1044 elements). (Right) Comparison of the central zone of the $1044(a), 1772$ (b) and 4544 (c) element meshes.

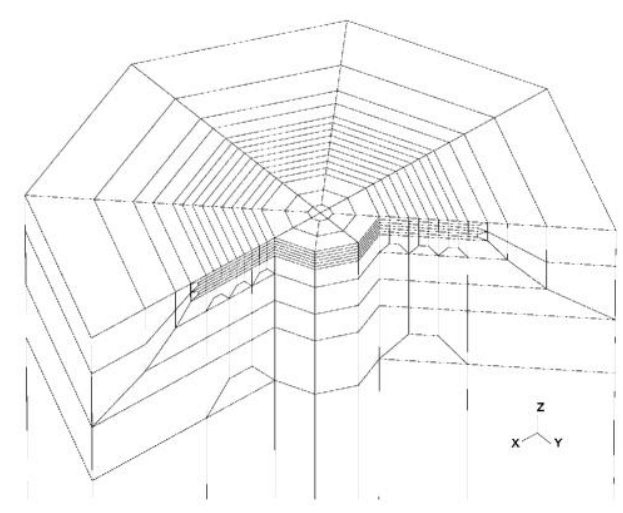

(b)

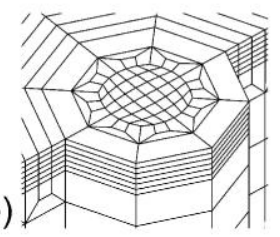

(a)

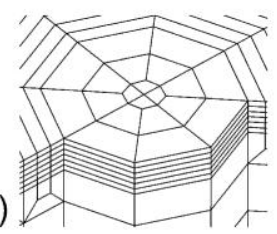

(c)

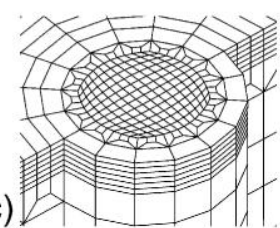




\section{Sensitivity analysis}

\subsection{Sensitivity to the mesh refinement and choice of one mesh}

In any FE calculation, the discretization chosen is a compromise between the conflicting requirements of accuracy and computational cost-effectiveness. To choose the appropriate mesh that reaches this compromise, numerical simulations with the three meshes presented above (Fig. 1) were performed, using the crystalline plasticity-based constitutive law with the PAN model and the parameters of Table 2. Corresponding load vs. displacement nanoindentation curves are presented in Fig. 2, where the experimental curve is plotted. With a small number of elements in the indented zone, bumps in the loading curve were more pronounced. Similarly, Liu et al. [15] observed that, with a coarser mesh, more and larger oscillations appear in the load vs. displacement curve. As mentioned in Gerday et al. [37], these bumps can be linked to the new integration points that enter into contact. For some authors [23], these oscillations in the numerical nanoindentation curves reflect model errors associated with the computer simulation of the moving contact. Nevertheless, all these comments agree with the same conclusions. When the mesh refinement is increased, the oscillation amplitude in the numerical load vs. displacement nanoindentation curves is reduced and the response tends to a smoother curve.

In Fig. 2, it appears that the number of elements in the FE mesh also influences the maximum load obtained for an imposed indentation depth. The maximum load is reduced when the number of elements increases. Contact is better localized when more degrees of freedom allow the material surface to follow the indent shape more closely.

For the following numerical nanoindentation simulations of this study, the second mesh is often used because it corresponds to a good compromise between accuracy and calculation cost.

\subsection{Sensitivity to the geometry of the indenter tip and validation of one tip}

In an ideal case, the experimental Berkovich indenter tip should be perfectly sharp; in practice, however, tips always present some curvature. Consequently, in this section, the influence of a small geometrical defect of the tip (curvature, included angle) on nanoindentation results is studied. To this end, numerical simulations were performed using the simple EP constitutive law and the parameters of Table 1.

First, the included angle of the Berkovich indenter is studied. A numerical simulation with the usual values of the total included angle and another one with a slightly more open angle were performed. The results are shown in Fig. 3. An increase of about 6\% in the included angle induces an increase of more than $30 \%$ in the maximum load, for the same indentation depth. This increase in the load for the same indentation depth using an increased opening angle was also observed by Yan et al. [14].

To understand the influence of the curvature always present at the bottom of the indenter, three different tip geometries were tested. In the FE simulations, the tip is modeled by rigid triangular facets. The first tip (A) is composed of three facets defined by four nodes (Fig. 4). The edges of this indenter are supposed to be perfectly planar without any curvature of the tip. The second one (B) is composed of nine facets defined by seven nodes. The three additional nodes are used to change the slope of the edges near the bottom of the tip in order to better simulate the natural curvature of the tip. The third one (C) is composed of 15 facets defined by 10 nodes. The three new nodes are also added to better simulate the curvature. Here, two changes in the slope of the edges are present near the bottom of the tip to smooth the geometrical variations compared to the perfect and nonrealistic tip (A). These tip geometries and the corresponding numerical results are presented in Fig. 4. From the load vs. displacement results obtained with these three tips, it appears that a decrease in the sharpness of the simulated indenter tip provides an increase in the maximum load, for the same indentation depth. Pelletier et al. [17] has also shown the necessity of taking the curvature into account to better simulate the experimental results.

Consequently, the next simulations use an indenter tip with a theoretical included angle of a Berkovich indenter $\left(142.3^{\circ}\right)$ and also model the tip curvature (tip (C) of Fig. 4). 
Fig. 2: Numerical nanoindentation simulations with three different meshes and the experimental result.

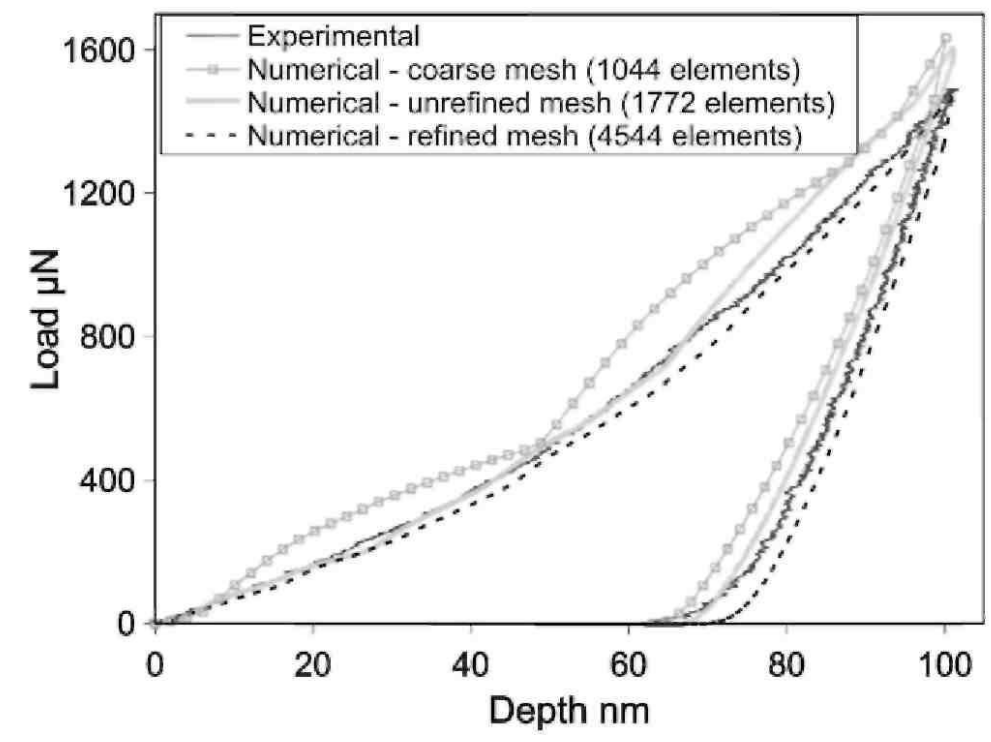

Fig. 3: Influence of the opening angle of the tip on the nanoindentation simulation results.

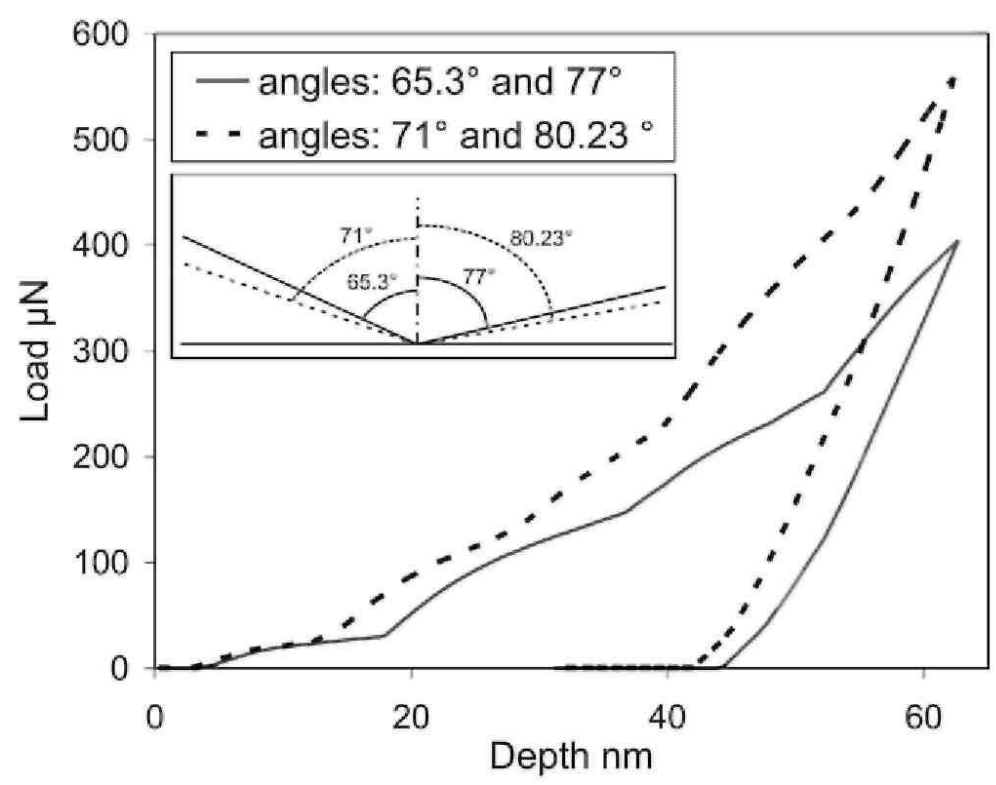


Fig. 4: Influence of the curvature of the tip on the nanoindentation simulation results. Tip without curvature (A), tip with three additional nodes $(B)$, and tip with six additional nodes $(C)$.

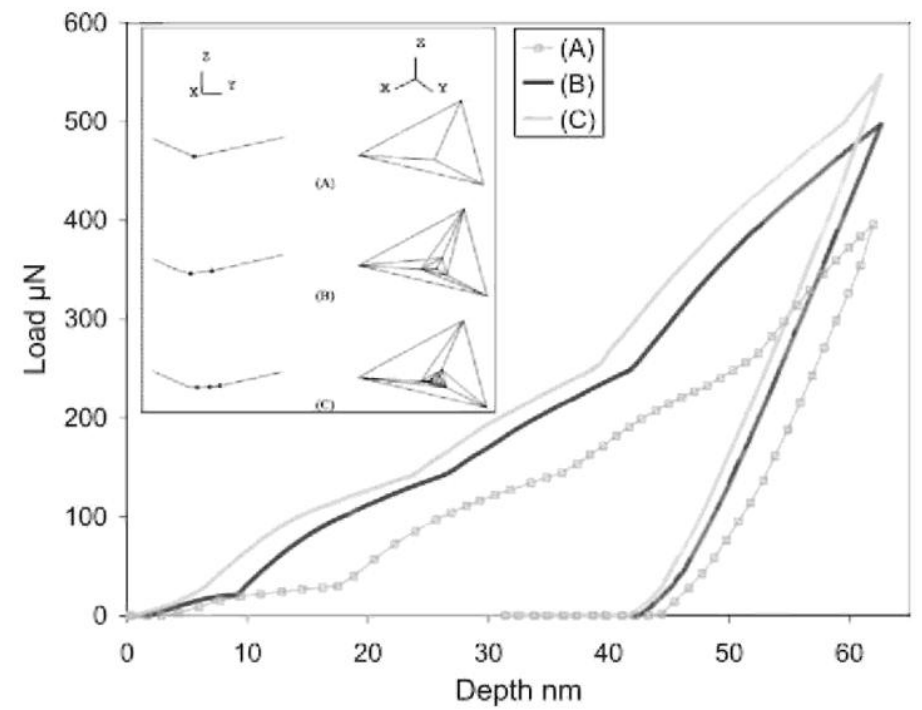

\subsection{Sensitivity to elastic parameters}

In this section, the effect of Young's modulus (Fig. 5a) and Poisson's ratio (Fig. 5b) are studied. Numerical simulations with the EP constitutive law were performed using different values of these parameters.

Young's modulus does not have to be known accurately because its influence on the increasing part of the load vs. displacement curve is limited. However, a sensible value of this parameter is important to prevent too large an error. Moreover, the value of Young's modulus strongly influences the slope of the unloading part of the nanoindentation load vs. displacement curve. Indeed, as shown in Fig. 5a, using a Young's modulus of 70 or 90 GPa leads to a change of $20 \%$ in the slope of the unloading nanoindentation curves.

On the other hand, a rough estimate of Poisson's ratio is enough to simulate this type of test. Poisson's ratio has a very small effect on the numerical nanoindentation curves (Fig. 5b). This result was also reported by Farrissey et al. [20], who observed that, over a reasonable range of Poisson's ratio values for metals, between 0.27 and 0.37 , the load vs. displacement indentation curves displayed only a small variation of less than $3 \%$ of the maximum indentation load.

\subsection{Sensitivity to the tangent modulus and the yield strength}

This section focuses on the usual plastic parameters: $E_{T}$, which represents the slope of the plastic part of the stress vs. strain curve; and $\sigma_{y 1}$, which is linked to plasticity onset. Nanoindentation simulations obtained with the EP constitutive law showing the influence of both parameters are presented in Fig. 6. It appears that a multiplication by a factor of 2 of the tangent modulus hardly affects the nanoindentation curve. On the other hand, when the yield strength is modified, changes in the load for the same indentation depth are important. In their study, Farrissey et al. [20] performed simulations of indentation using a series of yield strengths. The influence they report is close to the one observed in Fig. $6 \mathrm{~b}$.

\subsection{Sensitivity to the friction coefficient}

The role of the friction coefficient, $\mu$, on the load vs. displacement nanoindentation curves, was also studied. The results presented in Fig. 7 were obtained with the microscopic crystal plasticity-based constitutive law and friction coefficients from 0.02 to 0.5 . For better visibility, only a few of obtained results are shown in Fig. 7. As mentioned previously by Wang et al. [6] and Liu et al. [15], the friction coefficient has nearly no effect on numerical nanoindentation curves. This result was explained by Tabor [24] and is briefly mentioned again here. Friction between the indenter and the surface of the material is modeled by a Coulomb's law with a friction coefficient $\mu$ (Fig. 8). 
Fig. 5: Influence of Young's modulus (a) and Poisson's ratio (b) on indentation curves.
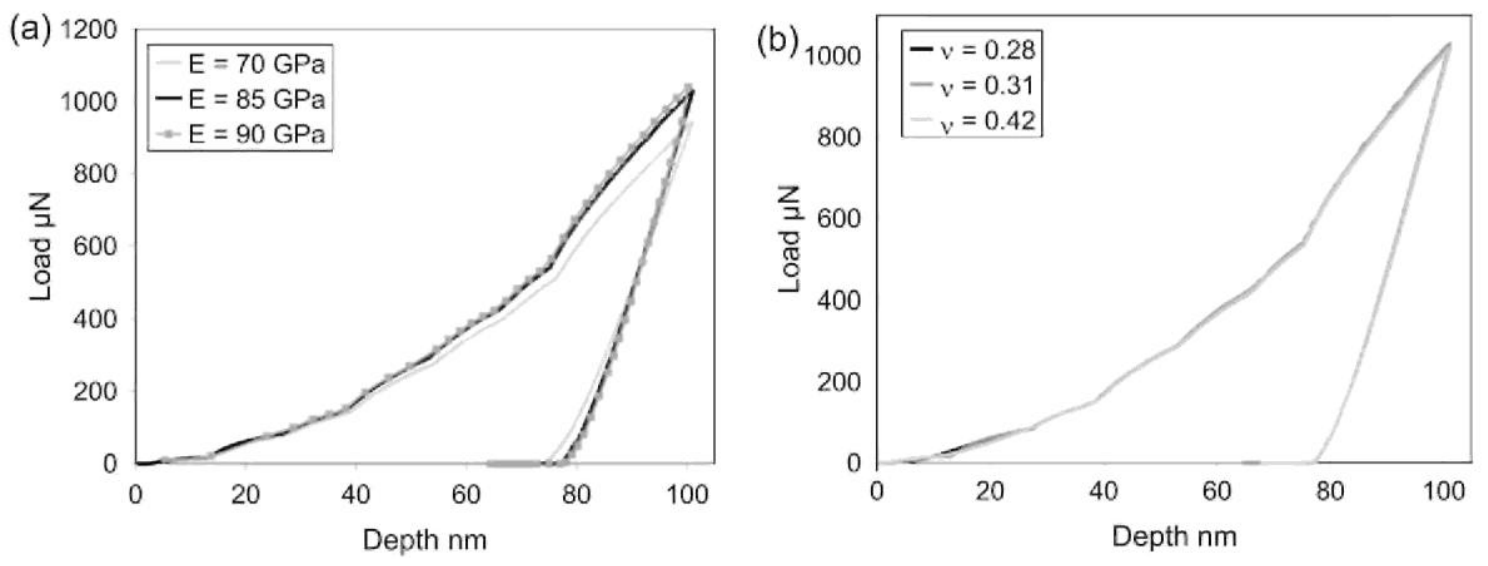

Fig. 6: Influence of the tangent modulus (a) and the plastic strength $(b)$ on nanoindentation curves.
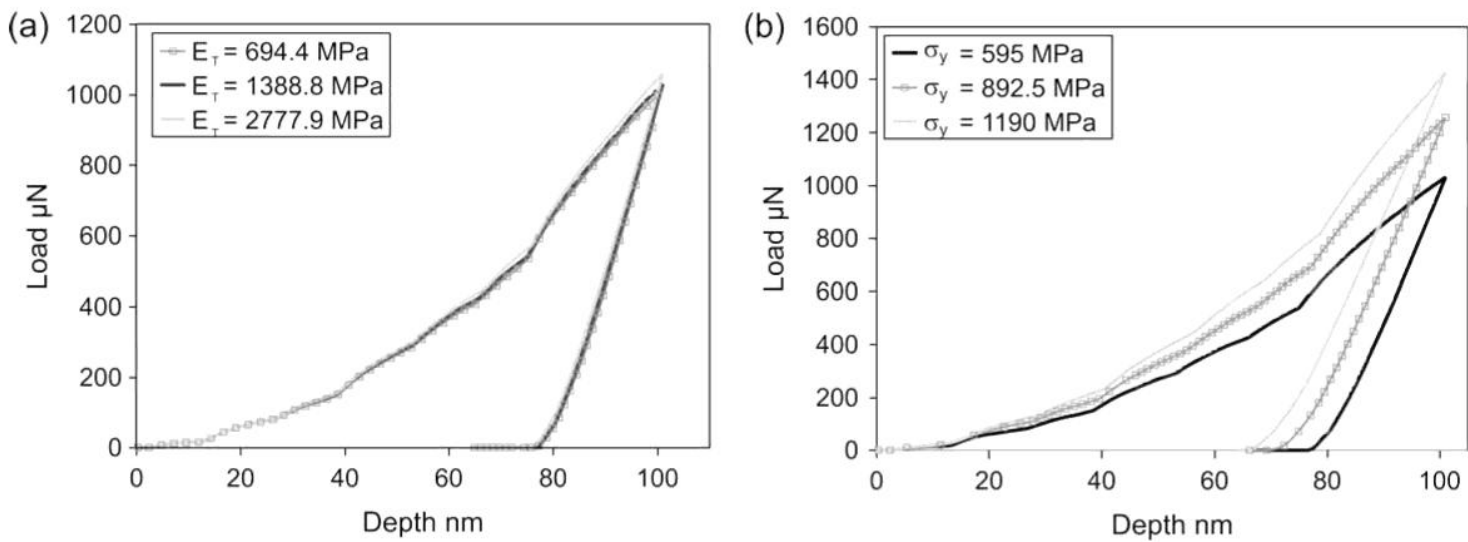

Therefore, the shear stress $\tau$ is equal to $\mu p$, where $p$, the contact pressure, is assumed to be constant at the interface and independent of the shape of the indenter. The normal force is equal to:

$F=\pi p R_{c}^{2}\left(1+\frac{\mu}{\tan \theta}\right)$

where $R_{\mathrm{c}}$ is the contact radius. Without friction, the contact pressure is equal to the ratio of the force to the projected contact area and is independent of the cone angle. For a Berkovich indenter, which is equivalent to a conical indenter with an included angle $\theta=70.3^{\circ}$ [22], the friction part, $\mu / \tan \theta$, of Eq. (12) is insignificant compared to the factor of 1 for usual values of the parameter $\mu$. Consequently, looking at the nanoindentation curve, the friction coefficient can be neglected in the case of Berkovich indentation.

However, the computation time varies greatly from one friction coefficient to another. For two numerical simulations performed with identical parameters apart from the friction coefficient, the computation time is about 25 times greater when a friction coefficient of 0.02 is chosen compared to a coefficient of 0.5 (due to convergence problems). 
Fig. 7: Influence of the friction coefficient on nanoindentation curves.

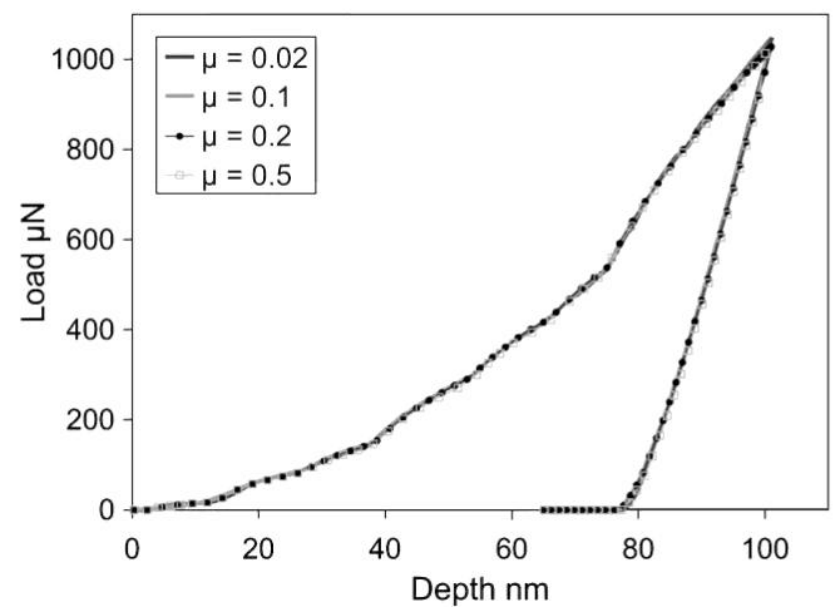

Fig. 8: Schematic drawing of a conical indentation, $\tau$ and $p$ represent the tangential and normal stresses, respectively, at each contact point.

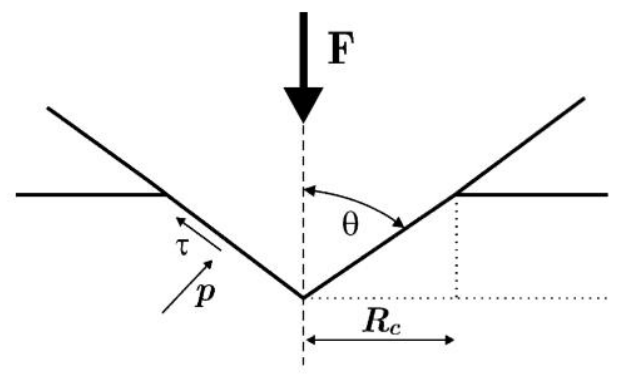

Moreover, some authors [15,22] have found that, while the introduction of friction does not change the nanoindentation load vs. displacement relationship, it changes the indent surface pile-up profiles. Friction prevents the material from piling-up at the interface. An increase in the friction coefficient leads to a reduction in the height of the pile-up patterns. Liu et al. [15] observed this phenomenon up to a friction coefficient of $\mu=0.4$. For greater values of this coefficient, no further reduction in pile-up height was perceived.

\subsection{Sensitivity to pile-up or sink-in patterns. Discussion of the literature, experimental and physical results}

Several authors have tried to predict the appearance of pile-up or sink-in patterns near the indented imprint and some of their conclusions are summarized here. According to Liu et al. [15], Bucaille et al. [22] and Bocciarelli et al. [23], a higher value of the friction coefficient leads to a reduction in the height of the pile-up patterns. Furthermore, other authors $[4,10,2,20]$ link the appearance of pile-up and sink-in patterns to hardening properties. For Xu et al. [2], who worked with elastic-perfectly plastic materials, a Young's modulus to plastic strength ratio $\left(E / \sigma_{y}\right)$ of 14.4 corresponds to a hard material, leading to sink-in patterns under indentation. An $E / \sigma_{y}$ ratio of 240 corresponds to a soft material for which pile-up patterns appear under indentation. In fact, for the same elastic modulus, a greater value of the yield stress will result in primarily elastic deformation. The indentation is accommodated by this far-field elastic displacement which results in sink-in patterns. On the other hand, a lower value of the yield stress will result in primarily plastic deformation. The material displaced by the indentation pushes out at the surface and forms a pile-up. Similarly to Xu et al. [2], also for elastic-perfectly plastic materials, Chen et al. [4] predict the appearance of pile-up or sink-in according to the $E / \sigma_{y}$ ratio, but in their case, the angle of a rigid cone used as the indenter also has an influence. Nevertheless, in practice, the appearance of pile-up patterns is not so easy to predict. As mentioned by Wang et al. [6], the pile-up or sink-in appearances are also due to the crystallography, i.e. the orientation of the indented grain.

In this section, simulations are performed using the two constitutive laws and different values of the $E / \sigma_{v}$ ratio. The goal of such simulations is to help understanding the appearance of pile-up or sink-in patterns in Ti-5553 alloy and finally to better reproduce the experimental results. Moreover, as explained in the previous section, validation of the friction coefficient should be possible by looking at the experimental and numerical patterns. 
Fig. 9: Indentation depth for different yield strengths and friction coefficients $(b)$ along a cut $(X=0)(a)$. Enlargement of the pile-up patterns of the soft material for the two friction coefficients (c).

(a)
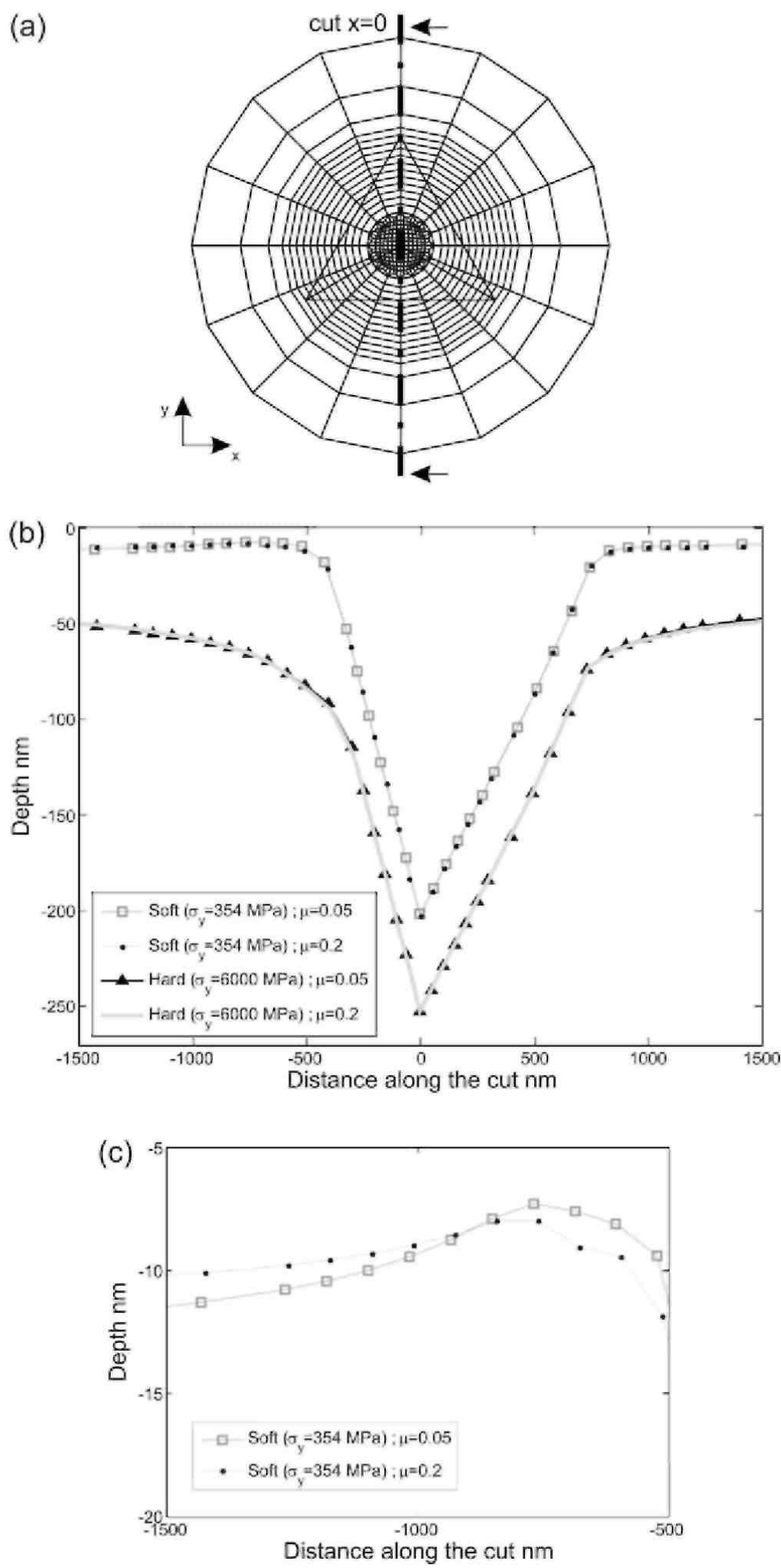


\subsubsection{Simulations with the simple EP constitutive law}

Simulations were performed using the simple EP constitutive law. For each value of the friction coefficient $(\mu=$ 0.05 and $\mu=0.2$ ), two values of $E / \sigma_{y}$ ratio were used: $E / \sigma_{y}=85,000 / 6000=14.2$ and $E / \sigma_{y}=85,000 / 354=240$. In Fig. 9, the shape of the indented surface is given for the four studied cases, for an indentation depth of 250 nm.

For $E / \sigma_{y}=240$, for which pile-up patterns are expected, very small ones are observed (Fig. 9c). Moreover, as was previously mentioned, the pile-up height is greater for a smaller friction coefficient. For $E / \sigma_{y}=14.2$, sink-in patterns appear, as expected.

\subsubsection{Simulations based on the crystalline constitutive law and experimental patterns}

Finally, two numerical simulations were performed using the crystalline constitutive law with the PAN hardening model. The parameters used are those given in Table 2 except for $\tau_{0}$ and $\tau_{\mathrm{s}}$, which are 30 and 35 , respectively, for the first simulation, and 3000 and 3500, respectively, for the second. The friction coefficient chosen is equal to 0.2 . The results obtained after a penetration depth of approximately $180 \mathrm{~nm}$ and before tip removal are given in Fig. 10.

Fig. 10: Indentation depth along a cut $(X=0)$, using the microscopic constitutive law and the PAN model.

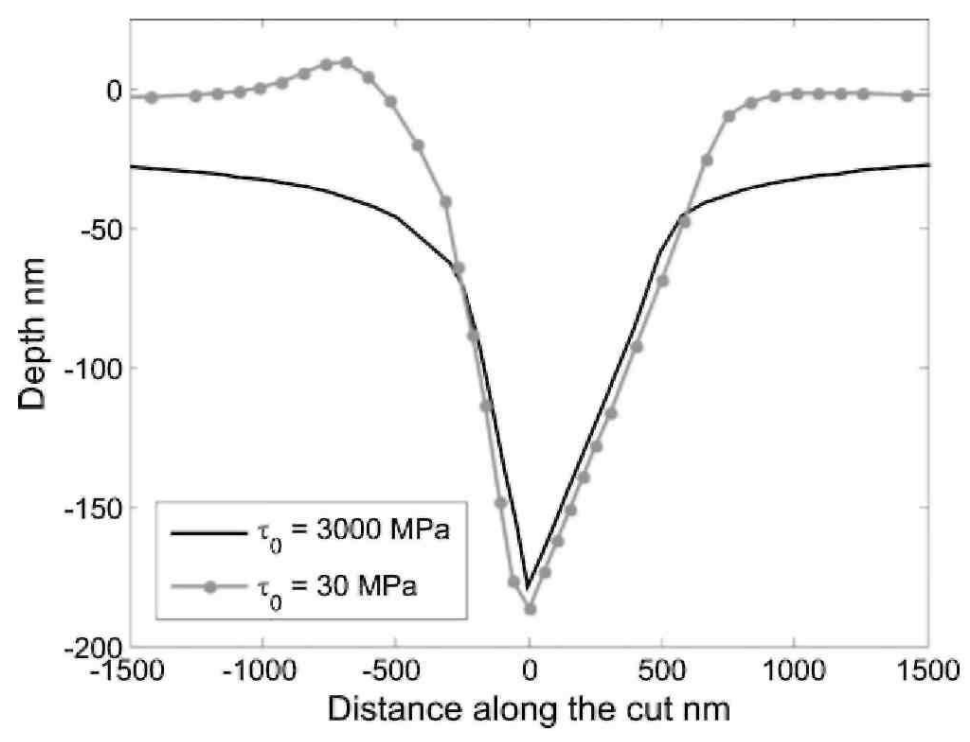

From literature results based on EP materials [4,2], pile-up patterns should appear in the simulation with low hardening (low $\tau_{0}$ ), when the material plastifies rapidly. Indeed, using a small value of the $\tau_{0}(=30 \mathrm{MPa})$ leads to pile-up patterns in the material around the imprint of the indenter tip.

For the simulation performed with a greater value of $\tau_{0}(=3000 \mathrm{MPa})$, the material should present sink-in patterns around the indent. This result is indeed observed.

Consequently, with such a law, it is possible to simulate pile-up patterns around the indented material and these are clearly more visible than with the EP constitutive law.

Fig. 11 shows a top view of the indented surface showing pile-up patterns and an electron backscattered diffraction (EBSD) image picture of the experimental $\beta$ material indented. Qualitatively, in each case, the size of the pile-up patterns is not the same along the three sides.

Unfortunately, due to computation time constraints, it was not possible to perform entire simulations (loading and tip removal) with this constitutive law using different friction coefficients in order to validate one value allowing close results between experimental and numerical patterns. 
Fig. 11: Contours of Z-displacement (in $10^{-8} \mathrm{~m}$ ) in the material indented after a penetration depth of approximately $180 \mathrm{~nm}$. Top view (a) and 3-D view (b) of the top of the material indented. Only the positive displacements are shown in the legend to visualize the pile-up patterns. Experimental pile-up patterns $(c)$.

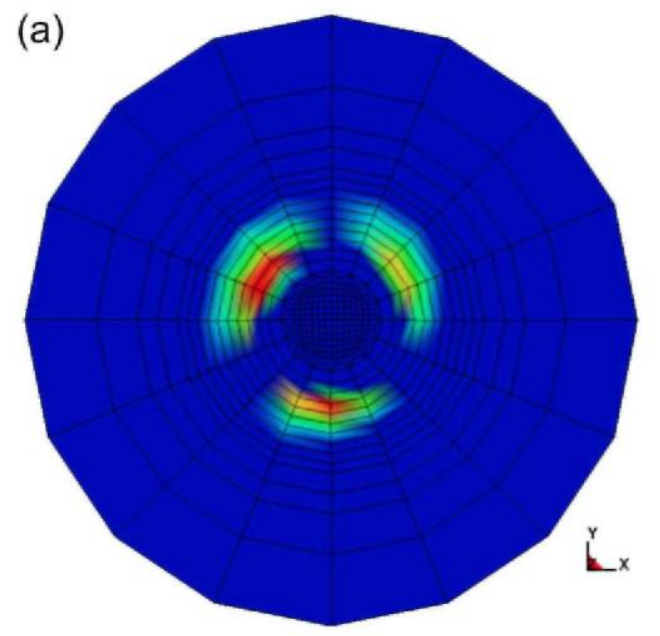

(b)

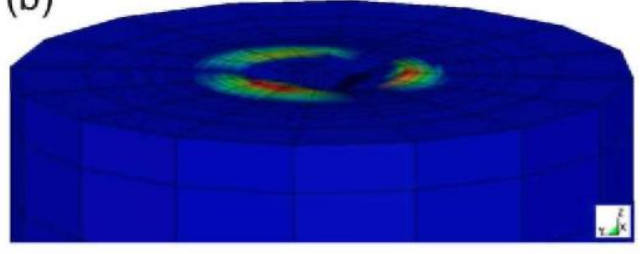

(c)

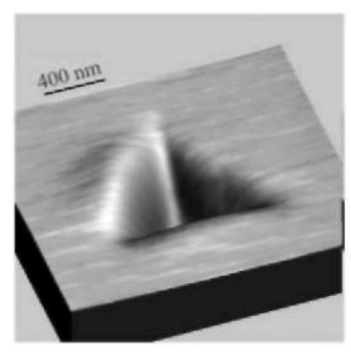

Contours of

-displacement

$4.441 \mathrm{e}-16$
$-1.786 \mathrm{e}+00$

$-3.572 e+00$

$-5.369 \mathrm{e}+00$

$.7 .145 \mathrm{e}+00$

$-8.931 \mathrm{e}+00$

$-1.072 e+01$ -

$-1.250 \mathrm{e}+01$ _

$-1.429 e+01$

$-1.608 \mathrm{e}+01$

$-1.786 e+01$

Fig. 12: Influence of the orientation of an indented bcc $\beta$ grain (of a Ti-5553 alloy) on load vs. displacement indentation curve. The orientation of the grains indented and their positions in the material are indicated.

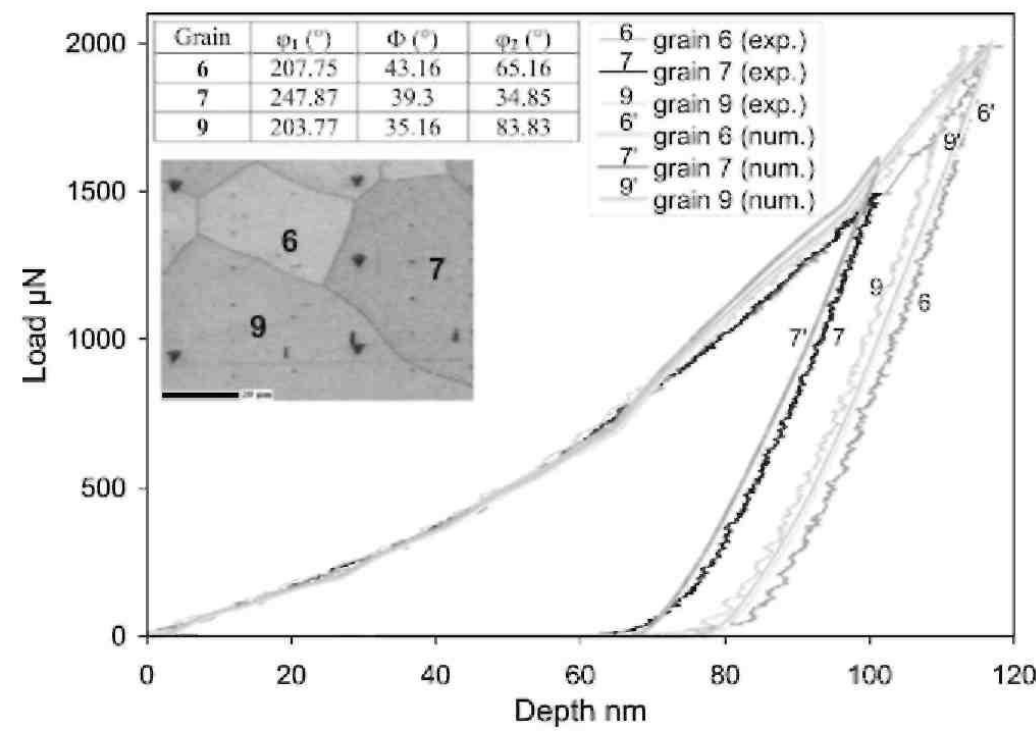




\subsection{Experimental validation from different grain orientations}

Finally, from a microscopic point of view, it is important to study the role of the grain orientation on load vs. displacement curves. Experimentally, it is possible to locate the grain indented and to measure its orientation using EBSD. Numerically, with the microscopic crystal plasticity-based constitutive law, the orientation of the grain is also taken into account. As a consequence, the numerical results obtained for a given grain orientation can be compared with the corresponding experimental ones.

Numerical nanoindentation simulations in the bcc $\beta$ phase of Ti-5553 were performed on different grain orientations using the microscopic constitutive law and the set of parameters optimized for the $\beta$ material (Table 2). The 1772 FE mesh and the indenter tip (C) in Fig. 4 were used for these numerical simulations. These nanoindentation tests should validate the set of parameters optimized for the $\beta$ phase. Results obtained for this validation are presented in Fig. 12, where experimental and numerical indentations in grains 6, 7 and 9 are compared. The orientations and location in the indented material of these three grains are given in Fig. 12.

The numerical nanoindentation results are in good agreement with experimental ones for the three grain orientations. The minor differences between the various grains are probably due to the large number of slip systems that can be activated in a bcc crystal. Thus, a rotation of the grain does not imply drastic modification of the behavior.

\section{Conclusions}

In this paper, a sensitivity analysis of several parameters on numerical nanoindentation tests was presented to determine the important material and geometrical parameters in a nanoindentation test and to validate the material parameters identified from macroscopic tests. We can draw the following conclusions:

- A fine mesh is necessary to avoid bumps in the nanoindentation curves. Thus, a compromise between accuracy and computation time was chosen. Moreover, the geometry of the tip must be modeled accurately to avoid excessive errors in the nanoindentation curve.

- The parameters that influence the unloading part of the nanoindentation curves are limited to the mesh refinement, the geometry of the indenter (the opening angle and the curvature) and the Young's modulus. An indenter tip with actual curvature modeling was validated.

- The Young's modulus has a limited effect on the loading phase and the Poisson's ratio has no effect on nanoindentation curves. Moreover, yield strength strongly influences the loading curve but the plastic modulus and friction coefficient do not significantly change the results.

- The difficulty obtaining pile-up patterns was presented. Parameters that generally influence the appearance and size of these patterns are the hardening parameters, the friction coefficient, the included angle of the indenter, and the orientation of the indented grain. It was found that the occurrence of pile-up or sink-in patterns is not so obvious. Using the EP constitutive law and appropriate choices of yield strength and friction coefficient, pile-up patterns appear but with a very small height. However, with the crystalline constitutive law and appropriate parameters, pile-up patterns become more visible.

- Finally, it was observed that the orientation of the indented bcc $\beta$ grain does not significantly influence the load vs. displacement nanoindentation curves. Moreover, these tests made the validation of the material parameters possible. Indeed, it appears that, using the parameters identified from macroscopic tests, gives nanoindentation results that are in good agreement with the experimental ones, for three different grain orientations.

\section{Acknowledgments}

The authors thank the Walloon Region (Titaero Project), the Belgian Scientific Research Fund FNRS which finances A.M.H. and L.D. and the Interuniversity Attraction Poles Program, Belgian Science Policy P6/24, for financial support. 
Published in: Acta Materialia (2009)

Status: Postprint (Author's version)

\section{References}

[1] Ahn JH, Jeon EC, Choi Y, Lee YH, Kwon D. Curr Appl Phys 2002;2:525-31

[2] Xu ZH, Li X. Acta Mater 2008;56:1399-405.

[3] Lilleodden ET, Nix WD. Acta Mater 2006;54:1583-93.

[4] Chen X, Vlassak JJ. J Mater Res 2001;16:2979-82.

[5] Pelletier H. Tribol Int 2005;39:593-606

[6] Wang Y, Raabe D, Klüber C, Roters F. Acta Mater 2004;52:2229-38.

[7] Larsson PL, Giannakopoulos AE, Söderlund E, Rowcliffe DJ, Vestergaard R. Int J Solids Struct 1996;33:221-48.

[8] Huang X, Pelegri AA. Compos Sci Technol 2007;67:1311-9.

[9] Saha R, Nix WD. Acta Mater 2002;50:23-38.

[10] Chen W, Li M, Zhang T, Cheng YT, Cheng CM. Mate Sci Eng A 2007;445-446:323-7.

[11] Doerner MF, Nix WD. J Mater Res 1986;1:601-9.

[12] Oliver WC, Pharr GM. J Mater Res 1992;7:1564-83.

[13] Sasaki T, Yang M, Fukushima S, Tsukano R. J Mater Process Tech 2004;151:263-7.

[14] Yan J, Karlsson AM, Chen X. Int J Solids Struct 2007;44:3720-37.

[15] Liu Y, Wang B, Yoshino M, Roy S, Lu H, Komanduri R. J Mech Phys Solids 2005;53:2718-41.

[16] Mirshams RA, Pothapragada RM. Acta Mater 2006;54:1123-34

[17] Pelletier H, Krier J, Cornet A, Mille P. Thin Solid Films 2000;379:147-55.

[18] Zaafarani N, Raabe D, Singh RN, Roters F, Zaefferer S. Acta Mater 2006;54:1863-76

[19] Liu Y, Varghese S, Ma J, Yoshino M, Lu H, Komanduri R. Int J Plast 2008;24:1990-2015.

[20] Farrissey LM, McHugh PE. Mater Sci Eng A 2005;399:254-66.

[21] Alcala J, Casals O, Ocenasek J. J Mech Phys Solids 2008;56:3277-303.

[22] Bucaille JL, Strauss S, Felder E, Michler J. Acta Mater 2003;51:1663-78.

[23] Bocciarelli M, Bolzon G, Maier G. Mech Mater 2005;37:855-68.

[24] Tabor D. The hardness of metals. Oxford: Clarendon Press; 1951

[25] Kojima Y, Shima M, Motoda T, Jibiki T, Sugawara T. Tribol Int 2007;40:1479-83.

[26] Gouldstone A, Chollacoop N, Dao M, Li J, Minor AM, Shen YL. Acta Mater 2007;55:4015-39.

[27] Dyduch M, Habraken AM, Cescotto S. Comput Method Appl Mech Eng 1992;101:283-98.

[28] Huang Y. Internal report. Cambridge (MA): Harvard University; 1991.

[29] Kysar JW. J Mech Phys Solids 2001;49:1099-128.

[30] Hutchinson JW. Proc Roy Soc Lond A Math Phys Sci 1976;348:101-27.

[31] Bassani JL, Wu T. Proc Roy Soc Lond A Math Phys Sci 1991;435:21-41.

[32] Siddiq A, Schmauder S, Huang Y. Int J Plast 2007;23:665-89. 
Published in: Acta Materialia (2009)

Status: Postprint (Author's version)

[33] Gerday AF. Ph.D. thesis. University of Liège; 2009.

[34] Gerday AF, Clement N, Jacques PJ, Pardoen T. Inverse modeling of nanoindentation tests to identify Ti-555 behavior. In: Proceedings of the Ti-2007 science and technology, Japan; 2007.

[35] Marquardt DW. SIAM J Appl Math 1963;11:431-40.

[36] Habraken AM, Cescotto S. Math Comput Model 1998;28:153-69.

[37] Gerday AF, Clement N, Jacques PJ, Pardoen T, Habraken AM. FE simulations of nanoindentation in beta metastable Ti. In: Proceedings of the ESAFORM 2006, Poland; 2006. 